\title{
THE DIFFERENCE BETWEEN CONSECUTIVE \\ PRIME NUMBERS. IV
}

\section{R. A. RANKIN}

1. Introduction. Let $p_{n}$ denote the $n$th prime, and let $\theta$ be the lower bound of all positive numbers $\sigma_{0}$ such that no Dirichlet $L$-function $L(s, \chi)$ has a zero at $s=\sigma+i t$, where $\sigma>\sigma_{0}$. Write

$$
l=\liminf _{n \rightarrow \infty} \frac{p_{n+1}-p_{n}}{\log p_{n}} .
$$

The purpose of this paper is to combine the methods used in two earlier papers 1 in order to prove the following theorem.

THEOREM.

$$
l \leqq c(1+4 \Theta) / 5,
$$

where $c<42 / 43$.

The precise definition of $c$ is given by (18) and (8) below.

The theorem is an improvement on the result $l \leqq(1+4 \Theta) / 5$ obtained in II. It was shown in III that

$$
l \leqq d=0.966 \cdots<57 / 59 \text {. }
$$

The numbers $c$ and $d$ are connected by the relation

$$
1-d=\left(3+2^{3 / 2}\right)(1-c) / 4>1-c,
$$

so that (1) is an improvement on (2) only if $\Theta$ is not too close to unity, in fact, if $\Theta<0.986 \ldots$. In particular, if the "grand Riemann hypothesis" is true, that is, if $\Theta=1 / 2$, we have (using the value of $c$ given by (18))

$$
l \leqq 3 c / 5<109 / 186=0.58602 \cdots .
$$

2. Notation. Since (2) is sharper than (1) for $\Theta=1$, we shall assume that $\Theta<1$, and write

$$
\sigma=(1+4 \Theta) / 5 .
$$

Let $N$ be a large positive integer, and define

$$
X=N^{1-\sigma}(\log N)^{-8(1+\gamma)}
$$

Received by the editors January 4, 1949.

1 See [2] and [3]; I refer to these as II and III respectively. Numbers in brackets refer to the references cited at the end of the paper. 
as done in equation (6.7) of II; here $\boldsymbol{\gamma}$ is a positive constant which may be taken to be 2 .

Let $\tilde{\omega}(\nu)$ be the number of prime pairs $p, p^{\prime}$ which satisfy

$$
N<p^{\prime} \leqq p \leqq 2 N, \quad p-p^{\prime}=\nu,
$$

where $\nu$ is an integer. We suppose that $h, k, m, n$ and $r$ are integers such that, for some positive $A_{1}, A_{2}$ independent of $N$,

$$
\begin{gathered}
A_{1} \log N<2 k / 3<h<k<A_{2} \log N, \\
0 \leqq m<n<A_{2} \log N, \\
r=k-h .
\end{gathered}
$$

The letters $a, c, \alpha, \beta$ denote positive numbers independent of $N$, and we write

$$
D=\prod_{p>2}\left(1+\frac{1}{p(p-2)}\right)
$$

and take

$$
\lambda=7.3566>e^{-2 \gamma} \Lambda_{0} / 2,
$$

where $\gamma$ is Euler's constant and $\Lambda_{0}$ is Buchstab's number 46.67347 (see relation (4) of III).

For $\mu=1,2, \cdots, k$ we define $a_{\mu}$ as follows:

$$
a_{\mu}=\left\{\begin{array}{rr}
\alpha & (0<\mu \leqq r, k-r<\mu \leqq k), \\
\beta & (r<\mu \leqq k-r),
\end{array}\right.
$$

and write

$$
\Psi(\theta)=\sum_{\mu=1}^{h} a_{\mu} e^{4 \pi 400}
$$

Then

$$
|\Psi(\theta)|^{2}=\xi(0)+2 \sum_{\nu=1}^{k-1} \xi(\nu) \cos 4 \pi \nu \theta,
$$

where

$$
\xi(\nu)=\sum_{i, j, 1 \leq j \leq i \leq k} a_{i} a_{j}
$$

\section{Lemmas.}

LEMMA 1. We have 


$$
\xi(\nu)=\left\{\begin{array}{lr}
2(r-\nu) \alpha^{2}+2 \nu \alpha \beta+(k-2 r-\nu) \beta^{2} & (0 \leqq \nu<r), \\
2 r \alpha \beta+(k-2 r-\nu) \beta^{2} & (r \leqq \nu<k-2 r), \\
(2 r-k+\nu) \alpha^{2}+2(k-r-\nu) \alpha \beta & (k-2 r \leqq \nu<k-r), \\
(k-\nu) \alpha^{2} & (k-r \leqq \nu<k),
\end{array}\right.
$$

and

$$
\sum_{v=1}^{k-1} \xi(\nu)=\frac{1}{2}\{2 r \alpha+(k-2 r) \beta\}^{2}+O(\log N) .
$$

This follows easily from (9) and (11), and we omit the proof. It is in this lemma that the restriction $k-r=h>2 k / 3$ made in (5) is used.

LEMMA 2.

$$
\begin{aligned}
\sum_{1 \leq q \leq x} \frac{\mu^{2}(q)}{\phi^{2}(q)} \sum_{f=0,(, q)-1}^{q-1} e^{d \pi i v f / q} \\
=\frac{2}{D} \prod_{p \mid p, p>2}\left(\frac{p-1}{p-2}\right)+O\left(\nu X^{-1}(\log \log X)^{2}\right) .
\end{aligned}
$$

Here $\mu(q)$ is the Möbius function, $\phi(q)$ is Euler's function, and $D$ is defined by (7).

It follows from the well known relation

$$
\sum_{f=0,(f, q)=1}^{q-1} e^{4 \pi i n f / q}=\sum_{d \mid(2 v, q)} d \mu\left(\frac{q}{d}\right)
$$

that

$$
\begin{aligned}
\sum_{1 \leq q \leq X} \frac{\mu^{2}(q)}{\phi^{2}(q)} \sum_{f=0,(f, q)=1}^{q-1} e^{4 \pi i v f / q}= & \sum_{q \leq X} \frac{\mu(q)}{\phi^{2}(q)} \prod_{p \mid(2 v, q)}(1-p) \\
= & \sum_{q=1}^{\infty} \frac{\mu(q)}{\phi^{2}(q)} \prod_{p \mid(2 v, q)}(1-p) \\
& -\sum_{q>X} \frac{\mu(q)}{\phi^{2}(q)} \prod_{p \mid(2 v, q)}(1-p) .
\end{aligned}
$$

By a straightforward reduction the first term on the right may be shown to equal

$$
\frac{2}{D} \prod_{p \mid p, p>2}\left(\frac{p-1}{p-2}\right)
$$


See, for example, $\$ 83.21-3.23$ of [1]. Since $1 / \phi(n)=O\left(n^{-1} \log \log n\right)$, the second term on the right is

$$
O\left\{X^{-1}(\log \log X)^{2} \prod_{p \mid 2 v}(p-1)\right\}=O\left\{\nu X^{-1}(\log \log X)^{2}\right\} .
$$

LEMMA 3. If $s \geqq 0$ and is independent of $N$, then

$$
\sum_{n=m}^{n-1} \nu^{\bullet} \prod_{p \mid v, p>2}\left(\frac{p-1}{p-2}\right)=D \sum_{v=m}^{n-1} \nu^{\bullet}+O\left\{(\log N)^{\bullet}(\log \log N)^{2}\right\} \text {. }
$$

The proof is similar to that of the lemma in III. We suppose that $d$ is any product $p_{i_{1}} p_{i_{2}} \cdots p_{i_{j}}$ of distinct odd primes, and let $d^{\prime}$ be the associated product

$$
d^{\prime}=\left(p_{i_{1}}-2\right)\left(p_{i_{2}}-2\right) \cdots\left(p_{i_{j}}-2\right) .
$$

We permit $d$ to take the value unity and then put $d^{\prime}=1$. Then, clearly,

$$
\begin{aligned}
\sum_{d<n} \frac{1}{d d^{\prime}} & =\sum_{d \geq 1} \frac{1}{d d^{\prime}}+O\left\{\frac{1}{n}(\log \log 3 n)^{2}\right\} \\
& =D+O\left\{\frac{1}{n}(\log \log 3 n)^{2}\right\},
\end{aligned}
$$

since $1 / d^{\prime}=O\left\{d^{-1}(\log \log d)^{2}\right\}$ for large $e^{2} d$; and

$$
\begin{aligned}
\prod_{p \mid r, p>2}\left(\frac{p-1}{p-2}\right) & =\prod_{p \mid p, p>2}\left(1+\frac{1}{p-2}\right) \\
& =\sum_{d \mid v} \frac{1}{d^{\prime}} .
\end{aligned}
$$

For a given $d^{\prime}, \nu$ is a multiple of $d$, say $\nu=\mu d$, and, since $m \leqq \nu<n, \mu$ takes all integer values in the interval $m / d \leqq \mu<n / d$, which we denote by $I(d)$. Clearly

$$
\sum_{\mu \in I(d)}(\mu d)^{\bullet}=\frac{n^{o+1}-m^{o+1}}{(s+1) d}+O\left(n^{0}\right),
$$

and

$$
\sum_{n=m}^{n-1} \nu^{0}=\frac{n^{0+1}-m^{0+1}}{s+1}+O\left(n^{0}\right)
$$

\footnotetext{
2 It is possible to dispense with the factor $(\log \log 3 n)^{2}$.
} 
Hence

$$
\begin{aligned}
\sum_{v=m}^{n-1} \nu^{0} \prod_{p \mid r, p>2} & \left(\frac{p-1}{p-2}\right)=\sum_{d<n} \frac{1}{d^{\prime}} \sum_{\mu \in I(d)}(\mu d)^{\circ} \\
= & \frac{n^{0+1}-m^{\circ+1}}{s+1} \sum_{d<n} \frac{1}{d d^{\prime}}+O\left(n^{\circ} \sum_{d<n} \frac{1}{d^{\prime}}\right) \\
= & \frac{n^{0+1}-m^{0+1}}{s+1}\left\{D+O\left(\frac{1}{n}(\log \log 3 n)^{2}\right)\right\} \\
& +O\left\{n^{\circ}(\log \log 3 n)^{2} \log 2 n\right\} \\
= & D \sum_{n=m}^{n-1} \nu^{\circ}+O\left\{n^{\circ}(\log 2 n)^{2}\right\},
\end{aligned}
$$

and the lemma follows from (6).

LEMMA 4.

$$
\begin{aligned}
\sum_{1 \leq q \leq x} \frac{\mu^{2}(q)}{\phi^{2}(q)} \sum_{f=0,(f, q)=1}^{q-1} & \sum_{v=1}^{k-1} \xi(\nu) e^{4 r i v f / q} \\
= & 2 \sum_{\nu=1}^{k-1} \xi(\nu)+O\left\{\log N(\log \log N)^{2}\right\} \\
= & \{2 r \alpha+(k-2 r) \beta\}^{2}+O\left\{\log N(\log \log N)^{2}\right\} .
\end{aligned}
$$

By Lemma 2, the left-hand member is equal to

$$
\frac{2}{D} \sum_{\nu=1}^{k-1} \xi(\nu) \prod_{p \mid v, p>2}\left(\frac{p-1}{p-2}\right)+o\left\{k X^{-1}(\log \log X)^{2} \sum_{\nu=1}^{k-1} \xi(\nu)\right\} \text {. }
$$

It follows from Lemmas 1 and 3 (with $s=0,1$ ) that this equals $2 \sum_{\nu=1}^{k-1} \xi(\nu)+O\left\{\log N(\log \log N)^{2}\right\}+O\left\{X^{-1}(\log N)^{3}(\log \log X)^{2}\right\}$, and the lemma follows from (4) and the second part of Lemma 1.

4. Proof of the theorem. By considering the expression

$$
I(N, k)=\int_{0}^{1}|S(\theta) \Psi(\theta)|^{2} d \theta,
$$

where

$$
S(\theta)=\sum_{N<p \leq 2 N} e^{2 \times 10 p} \log p \quad \quad(p \text { prime), }
$$


and $\Psi(\theta)$ is defined by (10), it is possible to show that, for large $N$,

$$
\begin{aligned}
& I(N, k)=\xi(0) N \log N\{1+o(1)\} \\
& +2\{1+o(1)\} \log ^{2} N \sum_{v=1}^{k-1} \xi(\nu) \tilde{\omega}(2 \nu) \\
& >N\{1+o(1)\} \sum_{1 \leq q \leq x} \frac{\mu^{2}(q)}{\phi^{2}(q)} \sum_{f=0,(f, q)-1}^{q-1}\left|\Psi\left(\frac{f}{q}\right)\right|^{2} \\
& +o\left(N \log ^{2} N\right) \\
& =\xi(0) N\{1+o(1)\} \sum_{1 \leq q \leq x} \frac{\mu^{2}(q)}{\phi(q)} \\
& +2 N\{1+o(1)\} \Re \sum_{1 \leq q \leq x} \frac{\mu^{2}(q)}{\phi^{2}(q)} \\
& \cdot \sum_{j=0,(f, q)=1}^{q-1} \sum_{\nu=1}^{k-1} \xi(\nu) e^{4 \pi i n / q}+o\left(N \log ^{2} N\right) .
\end{aligned}
$$

The proof of this is similar in every respect to the proof of (4.1) and (6.8) of II, except that $\Psi(\xi)$ is defined by (10) and is not $\sum_{v=1}^{k-1} e^{2 \pi i v \theta}$ as in II. The restriction $k<\log N$ made in II is unessential and can be replaced by $k<A_{2} \log N$.

It follows from (3), (4), (12), Lemma 4, and Lemma 4 of $^{3}$ II that

$$
\begin{aligned}
& 2\{1+o(1)\} \log ^{2} N \sum_{\nu=1}^{k-1} \xi(\nu) \tilde{\omega}(2 \nu) \\
&> 4 N\{1+o(1)\} \sum_{v=1}^{k-1} \xi(\nu)-\sigma N \log N\{1+o(1)\} \xi(0) \\
&+o\left(N \log ^{2} N\right) \\
&= 2 N\{2 r \alpha+(k-2 r) \beta\}^{2}\{1+o(1)\} \\
&-\sigma N \log N\left\{2 r \alpha^{2}+(k-2 r) \beta^{2}\right\}\{1+o(1)\} \\
&+o\left(N \log ^{2} N\right)
\end{aligned}
$$

Now, just as in III, it can be shown by Buchstab's form of Brun's method $^{4}$ that, for sufficiently large $N$,

'There is a misprint in the enunciation of the lemma which should read: "If $X$ is large $\sum_{1 \leqq \ell \leqq x} \mu^{2}(q) / \phi(q) \sim \log X . "$.

4 We must consider the interval $(N, 2 N)$ instead of the interval $(x / \log x, x)$. Our only reason for considering an interval of the form $(N, 2 N)$ is that it enables us to quote results directly from II. As far as Brun's method is concerned, the inequality stated remains true if we replace the left-hand side $\bar{\omega}(2 \nu)$ by the number of prime pairs $p, p^{\prime}$ which satisfy $A N<p^{\prime} \leqq p \leqq(A+1) N, p-p^{\prime}=2 v>0$ where $A \geqq 0$. 


$$
\tilde{\omega}(2 v)<\frac{4 \lambda N}{D(\log N)^{2}} \prod_{p \mid r, p>2}\left(\frac{p-1}{p-2}\right),
$$

where $\lambda$ is defined by (8). It follows from Lemmas 1 and 3 that

$$
\begin{aligned}
\sum_{\nu=k-r}^{k-1} \xi(\nu) \tilde{\omega}(2 r) & <\frac{4 \lambda N}{(\log N)^{2}} \sum_{v=k \rightarrow r}^{k-1} \xi(\nu)+O\left\{\frac{N}{\log N}(\log \log N)^{2}\right\} \\
& =\frac{2 \lambda \alpha^{2} r^{2} N}{(\log N)^{2}}+O\left\{\frac{N}{\log N}(\log \log N)^{2}\right\}
\end{aligned}
$$

since $r=O(\log N)$.

Accordingly, if we make the assumption that $\tilde{\omega}(2 \nu)=0$ for all $\nu<h=k-r$ we have, from (13) and (14), that

$$
\begin{aligned}
\left\{\frac{2 r \alpha+(k-2 r) \beta}{\log N}\right\}^{2} & \{1+o(1)\} \\
& -\frac{1}{2} \sigma\left\{\frac{2 r \alpha^{2}+(k-2 r) \beta^{2}}{\log V}\right\}\{1+o(1)\} \\
< & 2 \lambda \alpha^{2}\left(\frac{r}{\log N}\right)^{2}\{1+o(1)\} .
\end{aligned}
$$

We now take $\alpha=1, \beta=2^{1 / 2}$, and put

$$
\begin{aligned}
& k=\left[\frac{1}{2} \sigma\left(1+\frac{2^{1 / 2} a}{\lambda}\right) \log N\right], \\
& h=\left[\frac{1}{2} \sigma\left(1-\frac{a}{\lambda}\right) \log N\right],
\end{aligned}
$$

where $a$ is a fixed positive number satisfying

$$
a<3-2^{3 / 2} \text {. }
$$

It is easily verified that, with this choice of $a, h>2 k / 3$.

Then, for large $N$,

$$
r=\frac{a \sigma}{2 \lambda}\left(2^{1 / 2}+1\right)^{\circ} \log N+O(1),
$$

$$
\begin{aligned}
2 r \alpha+(k-2 r) \beta & =\left(2-2^{1 / 2}\right)\left(k+2^{1 / 2} h\right)=\frac{1}{2^{1 / 2}} \sigma \log N+O(1), \\
2 r \alpha^{2}+(k-2 r) \beta^{2} & =2 h=\sigma\left(1-\frac{a}{\lambda}\right) \log N+O(1) .
\end{aligned}
$$


It follows from (15) that

$$
\frac{1}{2} \sigma^{2}-\frac{1}{2} \sigma^{2}\left(1-\frac{a}{\lambda}\right) \leqq 2 \lambda\left\{\frac{\sigma a\left(2^{1 / 2}+1\right)}{2 \lambda}\right\}^{2},
$$

that is, that

$$
a \geqq 3-2^{3 / 2} \text {, }
$$

which contradicts (17).

We conclude, accordingly, that $\bar{\omega}(2 \nu)>0$ for some integer $\nu$ satisfying

$$
\nu<\frac{1}{2} \sigma\left(1-\frac{a}{\lambda}\right) \log N \text {. }
$$

It follows from this inequality, and from (17), that

$$
l \leqq \sigma c
$$

where

$$
c=1-\left(3-2^{3 / 2}\right) / \lambda=0.97667 \cdots<\frac{42}{43},
$$

and this proves the theorem.

Note added 24 April 1949. Professor Erdös has informed me that considerable improvements have recently been made in Brun's method by A. Selberg and others; this will presumably mean that Buchstab's constant $\Lambda_{0}$, and consequently my constants $c$ and $d$ (see equations (2) and (18)), can be replaced by smaller values.

\section{REFERENCES}

1. G. H. Hardy and J. E. Littlewood, Partitio Numerorum III: On the expression of a number as the sum of primes, Acta Math. vol. 44 (1923) pp. 1-70.

2. R. A. Rankin, The difference between consecutive prime numbers, II, Proc. Cambridge Philos. Soc. vol. 36 (1940) pp. 255-266.

3. - The difference between consecutive prime numbers, III, J. London Math. Soc. vol. 22 (1947) pp. 226-230.

Clare College, Cambridge 